\title{
Turbulence and Cloud Angular Momentum
}

\author{
Andreas Burkert \\ Max-Planck-Institut für Astronomie, Königstuhl 17, D-69117 \\ Heidelberg, Germany \\ Peter Bodenheimer \\ UCO/Lick Observatory, Department of Astronomy and Astrophysics, \\ University of California, Santa Cruz, CA 95064, USA
}

\begin{abstract}
Numerical models of molecular cloud cores are obtained, with assumed Gaussian random velocity fields that are consistent with moderate turbulence. If the velocity power spectrum is taken to be $P(k) \sim k^{n}$, with $n=-4$ or -3 , then the constructed line-of-sight velocity maps on the plane of the sky show gradients that can be interpreted as rotation. Deduced values of angular velocity, angular momentum, and dimensionless rotational parameter $\beta$ are consistent with observations. The velocity gradient $\Omega$ of an individual core is not a good indicator of its intrinsic angular momentum $J / M$. However, the distribution of deduced angular momenta from a large sample of cores with different random velocity fields is close to the distribution of actual angular momenta of these model cores if one assumes $J / M=p \Omega R^{2}$ where $R$ is the core radius and $p$ must be determined from a Monte-Carlo study. For centrally condensed cores the standard value of $p=0.4$ overestimates the mean intrinsic angular momentum by a factor of 3 .
\end{abstract}

\section{Introduction-Observations}

Dense cores of molecular clouds $\left(n \approx 10^{4}-10^{5} \mathrm{~cm}^{-3}\right)$ are known to be the sites of star formation. The distribution of angular momentum among the various cores has important consequences for (a) whether a single star or a binary is formed, (b) the period distribution of binaries, (c) the distribution of sizes of protostellar disks and their structure, and (d) the properties of emerging planetary systems. Observational evidence for rotation consists of gradients in the line-of-sight velocity along cuts across the cores (Goodman et al. 1993; Barranco \& Goodman 1998). The observed gradients, for cores of about $0.1 \mathrm{pc}$ in radius, are about 1.5 $\mathrm{km} \mathrm{s}^{-1} \mathrm{pc}^{-1}$ which, if interpreted as rigid-body rotation, give $\Omega \approx 5 \times 10^{-14}$ $\mathrm{s}^{-1}$. For uniform-density cores the values of specific angular momentum and dimensionless parameter $\beta$ are

$$
\begin{array}{r}
J / M=0.4 \Omega R^{2} \approx 2 \times 10^{21} \mathrm{~cm}^{2} \mathrm{~s}^{-1} \\
\beta=\frac{E_{\text {rot }}}{\left|E_{\text {grav }}\right|} \approx 0.03
\end{array}
$$


Over the observed range of core sizes $(0.06 \mathrm{pc}-0.6 \mathrm{pc})$ the rotational parameters scale as $\Omega \propto R^{-0.4}, J / M \propto R^{1.6}$, and $\beta \approx$ constant.

The assumption of rigid-body rotation is customarily used not only for the interpretation of observations, but also for the initial conditions for theoretical calculations (e. g. review by Bodenheimer et al. 2000). However the supersonic line widths in molecular clouds, and in particular the line width - size relation (Larson 1981) have been interpreted in terms of turbulent motion. Furthermore, line profiles in molecular clouds are consistent with Gaussian velocity fields with a Kolmogorov spectrum (Dubinski, Narayan, \& Phillips 1995). If the line width - size relation is written $\sigma(\lambda) \propto \lambda^{q}$, where $\lambda$ is the size and $\sigma$ is the velocity dispersion, then recent work by Goodman et al. (1998) shows that on size scales larger than $0.1 \mathrm{pc}, q \approx 0.5$ while on smaller scales $q \approx 0$ with $\sigma \approx 0.13 \mathrm{~km}$ $\mathrm{s}^{-1}$. This value is somewhat subsonic for $\mathrm{T}=10 \mathrm{~K}$, indicating that substantial dissipation of turbulent energy has taken place on this scale. The turbulent motions in the cores may however still result in rotational properties that are quite a bit more complicated than uniform rotation. In order to further explore the connection between turbulence and rotation, we investigate the following questions:

How does angular momentum originate in turbulent molecular cloud cores (Goldsmith \& Arquilla 1985; Goodman et al. 1993; Dubinski et al. 1995)?

How well can we estimate intrinsic rotational properties of cloud cores, in particular the specific angular momentum, from maps of the line-of-sight velocity?

In particular, is the assumption of uniform rotation a good one for the estimation of the specific angular momentum of a particular core?

\section{Construction of Turbulent Core Models}

The models are based on the following assumptions:

Dense cloud cores result from turbulent clumps which have become gravitationally bound by the dissipation of their turbulent energy.

Starless dense cores are still in an early stage of contraction, so their velocity fields still contain the signature of the turbulent velocity field of the environment in which they formed. These turbulent velocities are, however, subsonic (Barranco \& Goodman 1998).

The velocity field can be described as a Gaussian random field. The Fourier components $\overrightarrow{\hat{v}}(\vec{k})$ are then completely specified by the power spectrum $P(k)$.

The velocity dispersion $\sigma$ in cloud cores scales with their size $\lambda$ as $\sigma \sim \lambda^{q}$, with $0 \leq q \leq 0.5$. In this case the power spectrum is $P(k) \sim k^{n}$, where $n=-3-2 q$, which gives $P(k) \sim k^{-3}$ for the smaller cores and $P(k) \sim k^{-4}$ for cores larger than $0.1 \mathrm{pc}$. Note that these values are not very different from the Kolmogorov spectrum $P(k) \sim k^{-11 / 3}$. 

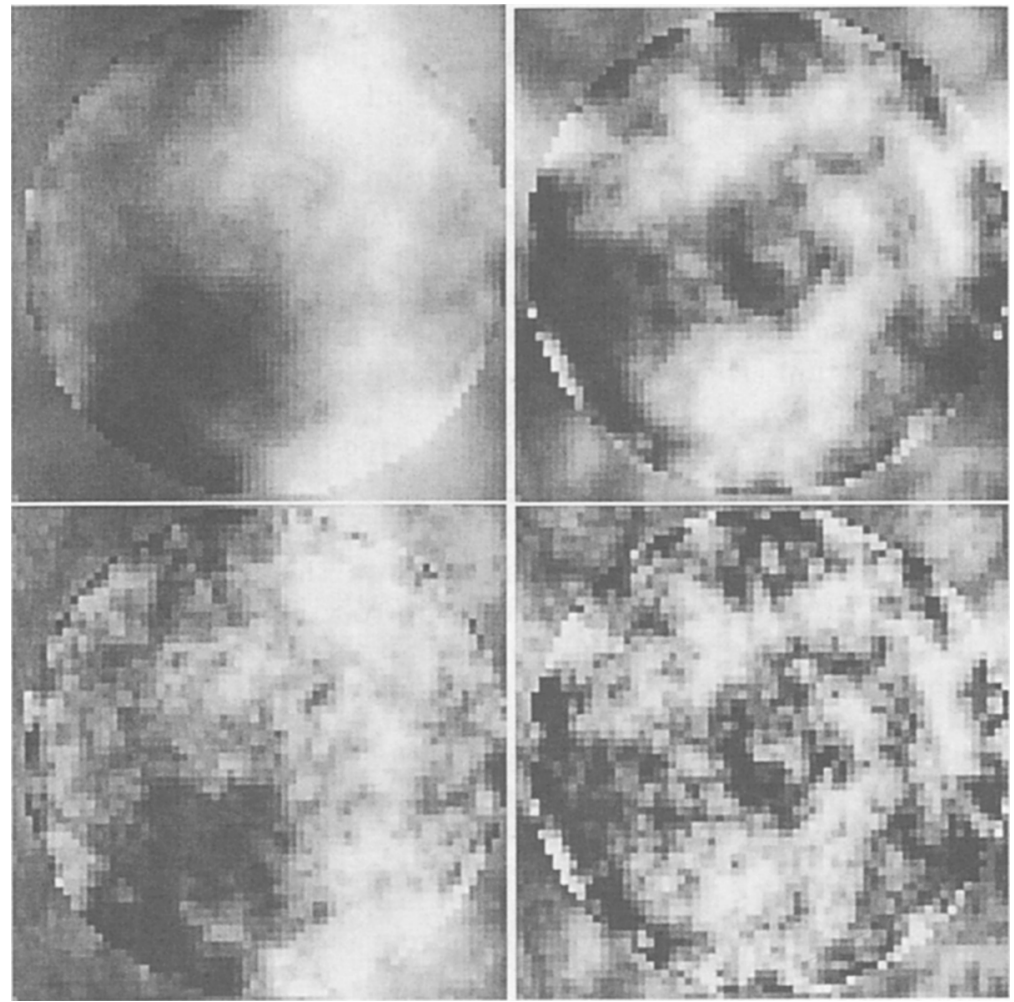

Figure 1. Examples of maps of dense cores of molecular clouds showing the normalized velocity in the line of sight for $n=-4$ (top row) and for $n=-3$ (bottom row), where $n$ describes the power spectrum of the random velocity field. Dark areas correspond to velocities toward the observer; light areas correspond to velocities away from the observer. In the top row, the values of intrinsic angular momentum $j$ are $0.9 \times 10^{21} \mathrm{~cm}^{2} \mathrm{~s}^{-1}$ (left) and $1.0 \times 10^{21} \mathrm{~cm}^{2} \mathrm{~s}^{-1}$ (right). The corresponding values of the angular velocity $\Omega$, deduced from the lineof-sight velocity gradient, are 1.9 and $0.2 \mathrm{~km} \mathrm{~s}^{-1} \mathrm{pc}^{-1}$. In the bottom row the values of $j$ are $0.4 \times 10^{21} \mathrm{~cm}^{2} \mathrm{~s}^{-1}$ (left) and $0.5 \times 10^{21} \mathrm{~cm}^{2} \mathrm{~s}^{-1}$ (right). The corresponding values of $\Omega$ are 0.7 and $0.06 \mathrm{~km} \mathrm{~s}^{-1} \mathrm{pc}^{-1}$. Each frame shows the projection of the full grid, but the velocity gradients and the intrinsic $j$-values are deduced from the region inside of which the density is at least half the maximum value. The radius of this region is assumed to be $0.1 \mathrm{pc}$. These examples show that the lineof-sight velocity gradient does in general not provide a good estimate of the intrinsic specific angular momentum. 
The initial density distribution is of Gaussian form, reasonably consistent with millimeter continuum observations of cores, which show them to be moderately centrally condensed (Ward-Thompson, Motte, \& André 1999):

$$
\rho(r)=\rho_{c} \exp \left(-3\left[\frac{r}{R_{\max }}\right]^{2}\right)
$$

where $\rho_{c}$ is the central density and $R_{\max }$ is the total radius.

The velocity field is uncorrelated with the spectral line emissivity.

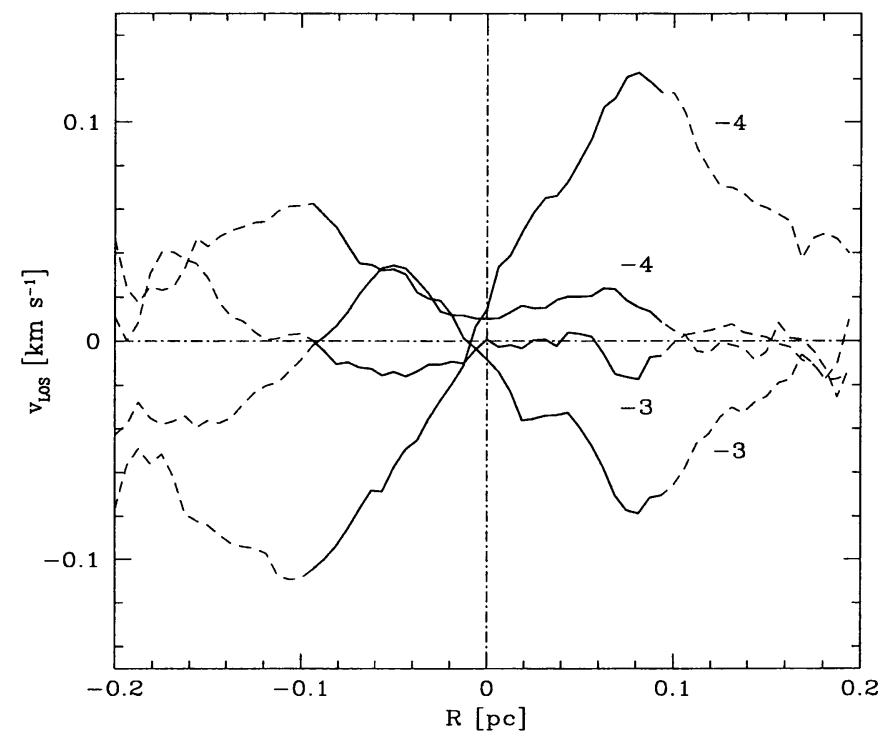

Figure 2. For the models shown in Fig. 1, the line-of-sight velocity, averaged over a strip parallel to the projected rotation axis, is plotted as a function of distance to the projected axis. The dashed lines indicate regions of low density that are not included in the analysis used for the determination of the velocity gradient. Each curve is labelled by the index $n$ of the power spectrum $P(k)$. Referring to the right-hand half of this figure, the upper and lower curves for $n=-4$ correspond, respectively, to the top left and top right frames of Fig. 1. The upper and lower curves for $n=-3$ correspond, respectively, to the bottom right and bottom left frames of Fig. 1 .

Models are constructed on a three-dimensional grid in Cartesian coordinates, with $N$ grid cells in each direction, where generally $N=64$. A Gaussian random velocity field with a given $P(k)$ is set up on this grid. Let the $(x, z)$ plane be the plane of the sky. By integrating along a set of lines of sight in the $y$ direction, one obtains a map of $N \times N$ pixels giving the density-weighted average line-of sight velocities. These maps can then be compared with observations; in practice only the inner part of the calculated cloud is used, corresponding to densities above the half-maximum value. With the exponential distribution given 

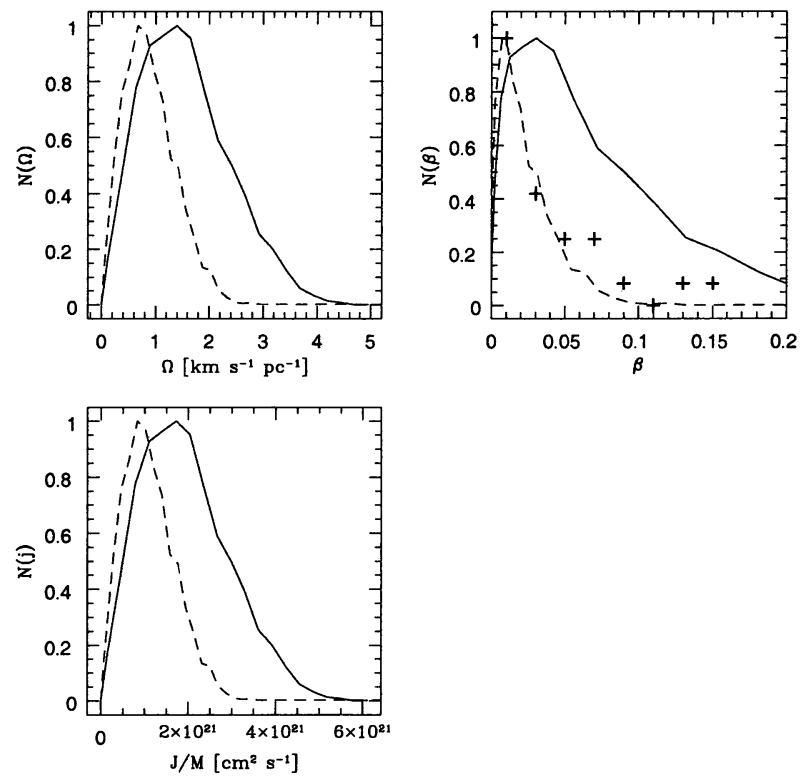

Figure 3. Results of 4000 random realizations of turbulent cores. Histograms for the angular velocity $\Omega$, as derived from the velocity gradient (top left), the specific angular momentum $j$ as derived from $\Omega$ (bottom), and the dimensionless rotational parameter $\beta$, as derived from $\Omega$ (top right). Solid curves: calculations with $n=-4$; dashed curves: calculations with $n=-3$. The observed distribution of $\beta$ values in cores (Goodman et al. 1993) is plotted in the top right frame.

above, the corresponding radius is about $0.5 R_{\max }$. Once a map has been obtained, the velocity gradient is determined and interpreted in terms of rotation, by minimizing the difference between the calculated line-of-sight velocity map and the map expected for a rigidly rotating cloud (Goodman et al. 1993; Burkert \& Bodenheimer 2000). The projected rotational velocity $\Omega$ is found, along with the angle of the projected rotation axis. In addition, the actual angular momentum of the 'observed' region is obtained by summation over the inner region of the full 3-D grid.

\section{Results}

\subsection{Unevolved Cores}

Here we consider cloud cores at an early stage when there are no collapse velocities, only a random Gaussian velocity field imposed on a fixed density distribution. Four examples of line-of-sight velocity maps are shown in Figure 1, two with $P(k) \propto k^{-4}$ and two with $P(k) \propto k^{-3}$. The two models illustrated in the top row have about the same intrinsic $j$, but the values of $\Omega$ derived from the velocity gradient differ by a factor of 10 . The same is true for the two models shown in the second row. Note also that for $n=-3$ the power on small scales increases, leading to more substructure and smaller intrinsic $j$. 
Figure 2 shows the deduced line-of-sight velocities as a function of projected distance to the rotation axis. It illustrates the fact that large velocity gradients arise if there are dominant long wavelength modes with small phase shifts relative to the center. This situation is more likely for the case $n=-4$ than for $n=-3$. Note that the left-hand frames of Figure 1 give well-defined velocity gradients within the 'observed' region, while the right-hand frames show practically no velocity gradient.

In order to derive physical quantities, we assume that a typical core with $R=0.1 \mathrm{pc}$ has an internal turbulent velocity dispersion $\sigma \approx 0.13 \mathrm{~km} \mathrm{~s}^{-1}$ and a mass $M \approx 5 \mathrm{M}_{\odot}$. Then from the value of $\Omega$ derived from the velocity gradient and assuming rigid-body rotation as in Goodman et al. (1993), we can obtain the corresponding specific angular momentum $J / M=0.4 \Omega R^{2}$ and the rotational parameter $\beta=\Omega^{2} R^{3} /(3 G M)$. Note that the equation for determining $J / M$ corresponds strictly only to uniform density cores and not to centrally condensed ones. Later, the derived $J / M$ will be compared with the intrinsic value.

For a given value of $n$ and a given density distribution, we then construct a set of 4000 line-of-sight velocity maps, each corresponding to a different random realization of the velocity field. A distribution of derived values of $\Omega, j$, and $\beta$ can thus be produced. The results are shown in Figure 3, for $n=-3$ and -4 . Averaged over these two distributions, the peaks fall at about $\langle\Omega\rangle \approx 1.0 \mathrm{~km}$ $\mathrm{s}^{-1} \mathrm{pc}^{-1},\langle j\rangle \approx 1.5 \times 10^{21} \mathrm{~cm}^{2} \mathrm{~s}^{-1}$, and $\langle\beta>\approx 0.02$, with a large spread in all quantities, assuming the cloud has a radius of $0.1 \mathrm{pc}$. These values are in reasonable agreement with observations, with better agreement for $n=-4$ than for $n=-3$. For cores of other sizes, one can derive scaling relations between the rotational parameters $j, \Omega, \beta$ and the global parameters $R, M, \sigma$ (Goodman et al. 1993):

$$
\Omega \sim \frac{\sigma}{R} ; j \sim \sigma \times R ; \quad \beta \sim \frac{\sigma^{2} R}{M} .
$$

If we adopt a line width-size relation $\sigma \sim R^{0.5}$ and virial equilibrium $\sigma^{2} \sim M / R$ we obtain

$$
j \sim \sigma R \sim R^{1.5} ; \quad \Omega \sim \frac{\sigma}{R} \sim R^{-0.5} ; \quad \beta \sim \sigma^{2} \frac{R}{M}=\text { const. }
$$

The results show that Gaussian random velocity fields with power spectra that are close to the Kolmogorov spectrum are in agreement with the line widthsize relation for molecular cloud cores and can explain observed gradients in the line-of-sight velocity. It is generally assumed that if the gradients are interpreted as rotation, then the specific angular momentum of the cloud can be determined. However in turbulent cores the specific angular momentum may not correlate with the deduced $\Omega$. This problem is illustrated in Figure 4, which, for a large sample of simulated cores and for $P(k) \propto k^{-4}$, shows that for a given $\Omega$, there can be a wide variety of values of the actual intrinsic angular momentum. The reason is that turbulent cores are not rigid rotators, and though they do contain a net angular momentum, compressional velocity components introduce velocity gradients that are not related to rotation. Thus the intrinsic $j$ of a core cannot be determined from a line-of-sight velocity map.

However, suppose we consider the distribution of angular momenta of a large sample of cores, all with the same $P(k)$. As $P(k)$ does not depend on the 


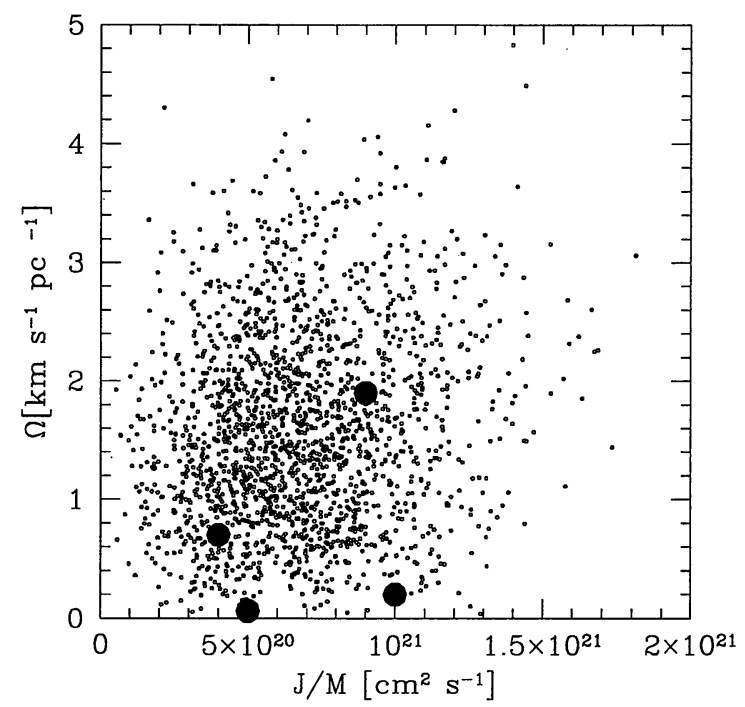

Figure 4. For a large sample of simulated cores $(n=-4)$, the projected $\Omega$ values are plotted as a function of the intrinsic specific angular momentum $j=J / M$. The large filled circles indicate the values of $j$ and $\Omega$ of the models illustrated in Fig. 1.

direction of $\vec{k}$, a set of maps of the line-of-sight velocity contains considerable information on the intrinsic distribution of angular momenta of the cores. Figure 5 , which illustrates a set of calculations for $n=-4$, compares the actual angular momenta of this set of cores (solid lines) with the angular momenta deduced from the derived velocity gradients and the formula $j=p \Omega R^{2}$, where $p$ is the dimensionless moment of inertia which depends on the density distribution of the core and is in general not $p=0.4$ as frequently adopted (Goodman et al. 1993). For both an exponential density distribution and a constant density the two curves for $j$ are in good agreement if $p=0.14$ in the former case and $p=0.4$ in the latter. Figure 5a illustrates the fact that for a centrally condensed core the use of $p=0.4$ gives an overestimate of the actual $j$-values by a factor of almost 3, while Figure 5b shows that for the constant-density case the actual dimensionless moment of inertia $p=0.4$ gives very good agreement between actual and deduced values of $j$.

\subsection{Evolved Cores}

The models in the previous section were calculated with a given assumed velocity distribution imposed on a fixed density profile. Thus the density structure is not entirely consistent with the velocity structure; however the profiles do become consistent if the model is evolved hydrodynamically. Here we discuss the early evolution of an initially turbulent core.

The starting point is a cloud of $1 \mathrm{M}_{\odot}$ with a uniform sound speed of $1 \times 10^{4}$ $\mathrm{cm} \mathrm{s}^{-1}$ with no ordered rotation and with a dimension $R_{\max }=1.088 \times 10^{17} \mathrm{~cm}$. The cloud is prolate with a density profile 

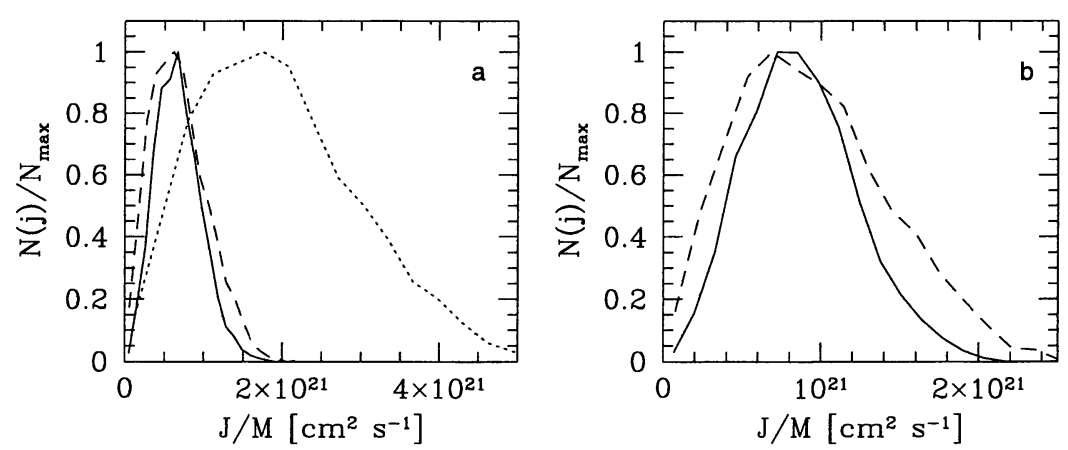

Figure 5. The distribution of specific angular momenta for simulated cores, as inferred from the projected velocity gradient and the relation $j=p \Omega R^{2}$ (dashed lines) is compared with the distribution of intrinsic specific angular momenta, as calculated from the 3-dimensional velocity field (solid lines). a): centrally condensed core. $b$ ): constant density core. The dotted line in frame $a$ gives the distribution of $j$ inferred from the velocity gradient with $p=0.4$, while the dashed line gives the distribution with an assumed $p=0.14$. In frame $b, p=0.4$ is used for the inferred values.

$$
\rho(r)=\rho_{c} \exp \left(-\frac{x^{2}}{a^{2}}-\frac{y^{2}+z^{2}}{b^{2}}\right)
$$

where the central density $\rho_{c}=6.2 \times 10^{-18} \mathrm{~g} \mathrm{~cm}^{-3}, a=R_{\max } / 1.73$, and $b=a / 2$. A turbulent velocity field with $P(k) \sim k^{-4}$ is imposed, giving a ratio of kinetic energy to gravitational potential energy of 0.3 and a ratio of internal energy to gravitational potential energy of 0.47 . The turbulent velocity field results in a net specific angular momentum $j=3.1 \times 10^{20} \mathrm{~cm}^{2} \mathrm{~s}^{-1}$. The isothermal evolution of the collapse is followed with an SPH code of 40,000 particles. As the collapse proceeds the turbulence gradually decays. The surface density distribution and line-of-sight velocity map viewed along the $z$-axis are shown in Figure 6 , at a time corresponding to two initial free-fall times at the cloud center; at this time some turbulence is still present, since the elapsed time is of order 0.1 times the sound crossing time of the initial configuration, and decay of turbulence takes place on a time scale of about one sound crossing time (Mac Low et al. 1998). In the density plot, there are two separated density maxima. In the velocity plot there is clear evidence of a velocity gradient, which, if interpreted as a rotation of the central region, would give $\Omega=3.0 \times 10^{-14} \mathrm{~s}^{-1}$. The velocity gradient determined for the same region at the initial stage gives almost the same value of $\Omega$. Thus the large-scale eddies which are responsible for the line-of-sight velocity gradients are still present even after an increase of the central density by a factor 3 . 

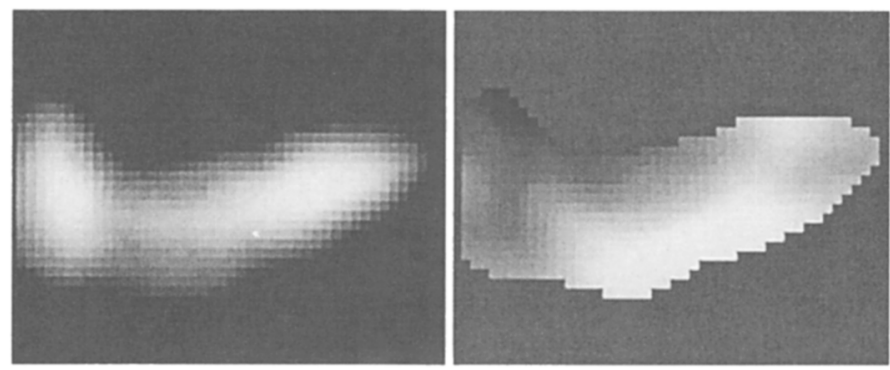

Figure 6. Map of surface density (left) and line-of-sight velocity (right) of the inner part of a collapsed core after a time of $7.8 \times 10^{11} \mathrm{~s}$. The highest mass density is $2.5 \times 10^{-17} \mathrm{~g} \mathrm{~cm}^{-3}$. Velocities range from $-0.05 \mathrm{~km} \mathrm{~s}^{-1}$ (dark) to $+0.07 \mathrm{~km} \mathrm{~s}^{-1}$ (light). The total width of the region plotted is $1.4 \times 10^{16} \mathrm{~cm}$. Only regions corresponding to densities greater than half the maximum are plotted.

\section{Conclusions}

Our simulations show that random Gaussian velocity fields with power spectra $P(k) \sim k^{-3}$ or $k^{-4}$ can reproduce both the observed line width-size relationship and the observed projected random properties of cloud cores. As cores are in general turbulent, such a velocity field should be used as an initial condition for more realistic simulations of core collapse and binary formation, instead of a rigidly rotating sphere ( $\mathrm{R}$. Klein \& $\mathrm{R}$. Fisher; this volume).

We find that cores that are statistically identical (i.e. generated with the same power spectrum) still show a large spread in their internal and deduced rotational properties. This spread is qualitatively in agreement with the large spread in observed binary periods and results from the random superposition of the various velocity modes.

The line-of-sight velocity gradients in general do not provide a good estimate of the internal specific angular momenta for a specific core. However, on a statistical basis, the distribution of projected velocity gradients can reproduce very well the distribution of internal specific angular momenta, if one assumes

$$
J / M=p \Omega R^{2}
$$

where $p$ depends on the density distribution of the core and has to be determined from a Monte-Carlo study. In general, adopting $p=0.4$ will overestimate the mean intrinsic angular momentum of condensed cores by a factor of 3 .

The linewidths in typical observed cores of molecular clouds indicate that the turbulence is mildly subsonic. No strong shocks are expected to form, and therefore the power spectrum in an evolved core should not be too different from the initial power spectrum in the core, as long as the evolution time is short 
compared with the decay time of the turbulence. Our analysis should therefore still remain valid in dynamically evolved cores. This conjecture has indeed been confirmed in a numerical calculation in which the dynamical evolution of the velocity field and the density field have been self-consistently determined.

\section{References}

Barranco, J. A., \& Goodman, A. A. 1998, ApJ, 504, 207

Burkert, A., \& Bodenheimer, P. 2000, ApJ, in press

Bodenheimer, P., Burkert, A., Klein, R. I., \& Boss, A. P. 2000, in Protostars and Planets IV, ed. V. Mannings, A. P. Boss \& S. Russell (Tucson: Univ. Arizona Press), 675

Dubinski, J., Narayan, R., \& Phillips, G. 1995, ApJ, 448, 226

Goldsmith, P. F., \& Arquilla, R. 1985, in Protostars and Planets II, ed. D. C. Black \& M. S. Matthews (Tucson: Univ. Arizona Press), 137

Goodman, A. A., Barranco, J. A., Wilner, D. J., \& Heyer, M. H. 1998, ApJ, 504,223

Goodman, A. A., Benson, P., Fuller, G., \& Myers, P. 1993, ApJ, 406, 528

Larson, R. B. 1981, MNRAS, 194, 809

Mac Low, M.-M., Klessen, R. S., Burkert, A. , \& Smith, M. D. 1998, Phys. Rev. Lett., 80, 2754

Ward-Thompson, D., Motte, F., \& André, P. 1999, MNRAS, 305, 143

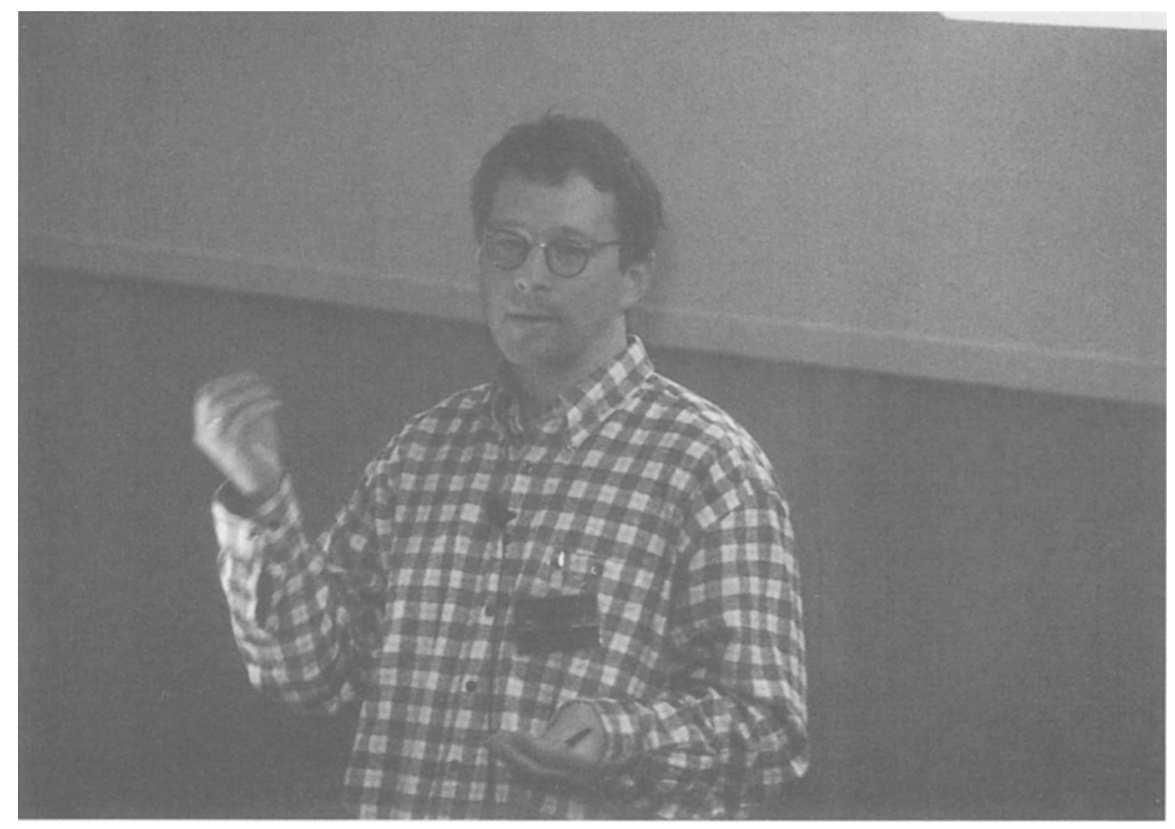

Andreas Burkert 